\title{
ON AN EXTENSION OF SINGULAR INTEGRALS ALONG MANIFOLDS OF FINITE TYPE
}

\author{
ABDELNASER Al-HASAN AND DASHAN FAN
}

Received 14 November 2005; Revised 6 April 2006; Accepted 25 April 2006

We extend the $L^{p}$-boundedness of a class of singular integral operators under the $H^{1}$ kernel condition on a compact manifold from the homogeneous Sobolev space $\dot{L}_{\alpha}^{p}\left(\mathbb{R}^{n}\right)$ to the Lebesgue space $L^{p}\left(\mathbb{R}^{n}\right)$.

Copyright (c) 2006 Hindawi Publishing Corporation. All rights reserved.

\section{Introduction}

Let $S^{n-1}$ be the unit sphere in $\mathbb{R}^{n}, n \geq 2$, with the normalized Lebesgue measure $d \sigma=$ $d \sigma\left(x^{\prime}\right)$. Let $\Omega\left(x^{\prime}\right)$ be a homogeneous function of degree 0 , with $\Omega \in L^{1}\left(\mathbf{S}^{n-1}\right)$ and

$$
\int_{\mathbf{S}^{n-1}} \Omega\left(x^{\prime}\right) d \sigma\left(x^{\prime}\right)=0
$$

where $x^{\prime}=x /|x|$ for any $x \neq 0$.

Suppose that $h$ is an $L^{\infty}\left(\mathbb{R}^{+}\right)$function; the singular integral operator $\mathrm{SI}_{\Omega, h}$ is defined by

$$
\mathrm{SI}_{\Omega, h}(f)(x)=\text { p.v. } \int_{\mathbb{R}^{n}} h(|y|) \frac{\Omega\left(y^{\prime}\right)}{|y|^{n}} f(x-y) d y
$$

for all test functions $f$, where $y^{\prime}=y /|y| \in \mathbf{S}^{n-1}$.

We denote $\mathrm{SI}_{\Omega, h}(f)$ by $\mathrm{SI}_{\Omega}(f)$ if $h=1$. The operator $\mathrm{SI}_{\Omega}$ was first studied by Calderón and Zygmund in their well-known papers (see $[1,2])$. They proved that $\mathrm{SI}_{\Omega}$ is $L^{p}\left(\mathbb{R}^{n}\right)$ bounded, $1<p<\infty$, provided that $\Omega \in L \log ^{+} L\left(\mathbf{S}^{n-1}\right)$ satisfying (1.1). They also showed that the space $L \log ^{+} L\left(\mathbf{S}^{n-1}\right)$ cannot be replaced by any Orlicz space $L^{\phi}\left(\mathbf{S}^{n-1}\right)$ with a monotonically increasing function $\phi$ satisfying $\phi(t)=o(t \log t), t \rightarrow \infty$, that is, $L\left(\log ^{+} L\right)^{1-\varepsilon}\left(\mathbf{S}^{n-1}\right), 0<\varepsilon \leq 1$. The idea of their proof was as follows.

Suppose that $\Omega \in L^{1}\left(\mathbf{S}^{n-1}\right)$ is an odd function, then one can easily show that

$$
\mathrm{SI}_{\Omega}(f)(x)=\frac{1}{2} \int_{\mathbf{S}^{n-1}} \Omega\left(y^{\prime}\right)\left\{\int_{-\infty}^{\infty} f\left(x-t y^{\prime}\right) t^{-1} d t\right\} d \sigma\left(y^{\prime}\right)
$$


By the method of rotation and the well-known $L^{p}$-boundedness of the Hilbert transform, one then obtains the $L^{p}$-boundedness of $\mathrm{SI}_{\Omega}$ under the weak condition $\Omega \in L^{1}\left(\mathbf{S}^{n-1}\right)$.

For even kernels, the condition $\Omega \in L^{1}\left(\mathbf{S}^{n-1}\right)$ is insufficient. It turns out that the right condition is $\Omega \in L \log ^{+} L\left(\mathbf{S}^{n-1}\right)$ (as far as the size of $\Omega$ is concerned). The idea of Calderón and Zygmund is to compose the operator $\mathrm{SI}_{\Omega}$ with the Riesz transforms $R_{j}$, $1 \leq j \leq n$, and to show that $R_{j}\left(\mathrm{SI}_{\Omega}\right)$ is a singular integral operator with an appropriate odd kernel. Thus

$$
\left\|R_{j}\left(\mathrm{SI}_{\Omega}\right)(f)\right\|_{p} \leq C_{p}\|f\|_{p}
$$

for all test functions $f \in \mathscr{Y}$. Furthermore, one can obtain

$$
\begin{aligned}
\left\|\mathrm{SI}_{\Omega}(f)\right\|_{p} & =\left\|\left(\sum_{j=1}^{n} R_{j}^{2}\right) \mathrm{SI}_{\Omega}(f)\right\|_{p} \leq \sum_{j=1}^{n}\left\|R_{j}\left(R_{j} \mathrm{SI}_{\Omega}(f)\right)\right\|_{p} \\
& \leq \mathrm{nC} \sum_{j=1}^{n}\left\|R_{j} \mathrm{SI}_{\Omega}(f)\right\|_{p} \leq n^{2} \mathrm{CC}_{p}\|f\|_{p}
\end{aligned}
$$

for all test functions $f \in \mathscr{Y}$, since $-\sum_{j=1}^{n} R_{j}^{2}$ is the identity map. Using the above method, Connett [7] and Ricci and Weiss [15] independently obtained the same $L^{p_{-}}$ boundedness of $\mathrm{SI}_{\Omega}$ under the weak condition $\Omega \in H^{1}\left(\mathbf{S}^{n-1}\right)$, where $H^{1}\left(\mathbf{S}^{n-1}\right)$ is the Hardy space which contains $L \log ^{+} L\left(\mathbf{S}^{n-1}\right)$ as a proper subspace.

In [12], Fefferman generalized this Calderón-Zygmund singular integral by replacing the kernel $\Omega\left(x^{\prime}\right)|x|^{-n}$ by $h(|x|) \Omega\left(x^{\prime}\right)|x|^{-n}$, where $h$ is an arbitrary $L^{\infty}$ function. This allows the kernel to be rough not only on the sphere but also in the radial direction. For the singular integral operator $\mathrm{SI}_{\Omega, h}$ with the kernel $K(x)=h(|x|)\left(\Omega\left(x^{\prime}\right) /\left(|x|^{n}\right)\right)$, the formula (1.3) now is

$$
\operatorname{SI}_{\Omega, h}(f)(x)=\int_{S^{n-1}} \Omega\left(y^{\prime}\right)\left\{\int_{0}^{\infty} f\left(x-t y^{\prime}\right) h(t) t^{-1} d t\right\} d \sigma\left(y^{\prime}\right) .
$$

Clearly, the method of Calderón and Zygmund can no longer be used to estimate the above integral in (1.6) even if $\Omega$ is odd, since the integral in parentheses cannot be reduced to the Hilbert transform for an arbitrary $h(t)$. Thus, one needs to find a new approach.

Using a method which is different from Calderón and Zygmund, Fefferman showed in [12] that if $\Omega$ satisfies a Lipschitz condition, then $\mathrm{SI}_{\Omega, h}$ is bounded on $L^{p}\left(\mathbb{R}^{n}\right)$ for $1<p<\infty$. Later in [8], using Littlewood-Paley theory and Fourier transform methods, Duoandikoetxea and Rubio de Francia improved Fefferman's results by assuming a roughness condition $\Omega \in L^{q}\left(\mathbf{S}^{n-1}\right)$ (see also $[3,13,14]$ ). By modifying the method in [8], recently, Fan and Pan [11] have improved the above results on $\mathrm{SI}_{\Omega, h}$ by assuming a roughness condition $\Omega \in H^{1}\left(\mathbf{S}^{n-1}\right)$. 
Noting that $S^{n-1}$ is an $(n-1)$-dimensional compact manifold in $\mathbb{R}^{n-1}$, Duoandikoetxea and Rubio de Francia [8] introduced the following extension of the operator $\mathrm{SI}_{\Omega, h}$.

Let $m, n \in \mathbb{N}, m \leq n-1$, and let $M$ be a compact, smooth, $m$-dimensional manifold in $\mathbb{R}^{n}$. Suppose that $\mathcal{M}\left\{\{r v: r>0\}\right.$ contains at most one point for any $v \in \mathbf{S}^{n-1}$. Let $\mathscr{C}(\mathcal{M})$ denote the cone $\{r \theta: r>0, \theta \in M\}$ equipped with the measure $d s(r \theta)=r^{m} d r d \sigma(\theta)$, where $d \sigma$ represents the induced Lebesgue measure on $M$. For a locally integrable function in $\mathscr{C}(\mathcal{M})$ of the form

$$
K(r \theta)=r^{-m-1} h(r) \Omega(\theta)
$$

where $\Omega$ satisfies

$$
\int_{\mathcal{M}} \Omega(\theta) d \sigma(\theta)=0
$$

they defined the corresponding singular integral operator $\mathrm{SI}_{\mathcal{M}, \Omega, h}$ on $\mathbb{R}^{n}$ by

$$
\begin{aligned}
\left(\mathrm{SI}_{\mathcal{M}, \Omega, h} f\right)(x) & =\mathrm{p} \cdot \mathrm{v} \cdot \int_{\mathscr{C}(\mathcal{M})} f(x-y) K(y) d s(y) \\
& =\lim _{\varepsilon \rightarrow 0^{+}} \int_{\mathcal{E}}^{\infty} \int_{\mathcal{M}} f(x-r \theta) \Omega(\theta) h(r) r^{-1} d \sigma(\theta) d r
\end{aligned}
$$

initially for $f \in \mathscr{S}\left(\mathbb{R}^{n}\right)$.

In [8], Duoandikoetxea and Rubio de Francia obtained the following results regarding $\mathrm{SI}_{\mathcal{M}, \Omega, h}$.

Theorem 1.1. Let $\mathrm{SI}_{\mathcal{M}, \Omega, h}$ be given as in (1.7)-(1.9). Suppose that

(i) $\Omega \in L^{q}(M)$,

(ii) $\sup _{R>0}\left((1 / R) \int_{0}^{R}|h(r)|^{2} d r\right)<\infty$,

(iii) $M$ has a contact of finite order with every hyperplane.

Then $\mathrm{SI}_{\mathcal{M}, \Omega, h}$ extends to a bounded operator on $L^{p}\left(\mathbb{R}^{n}\right)$ for $1<p<\infty$.

Inspired by the earlier result of Fan and Pan regarding $\Omega \in H^{1}\left(\mathbf{S}^{n-1}\right)$, Cheng and Pan [5] established the following.

Theorem 1.2. Let $\mathrm{SI}_{\mathcal{M}, \Omega, h}$ be given as in Theorem 1.1, and let $h$ and $M$ satisfy (ii) and (iii), respectively. If $\Omega \in H^{1}(\mathcal{M})$, then $\mathrm{SI}_{\mathcal{M}, \Omega, h}$ extends to a bounded operator on $L^{p}\left(\mathbb{R}^{n}\right)$ for $1<p<\infty$.

The main purpose of this paper is to extend Theorem 1.2 to the case $\Omega \in H^{r}(\mathcal{M})$ with $0<r<1$. The space $H^{r}(\mathcal{M})$ is a distribution space when $0<r<1$. The definition of $H^{r}(M)$ can be found in Section 2, but here we must define the operator in the sense of distribution. 
4 Singular integrals along manifolds

Let $\langle\Omega, \phi\rangle$ be the pairing between $\Omega \in H^{r}(\mathcal{M})$ and a $C^{\infty}$ function $\phi$ on $M$. For $0 \leq \alpha$, we define the singular integral operator $\mathrm{SI}_{\mathcal{M}, \Omega, h, \alpha} f(x)$ by

$$
\operatorname{SI}_{M, \Omega, h, \alpha} f(x)=\lim _{\varepsilon \rightarrow 0^{+}} \int_{\varepsilon}^{\infty}\langle f(x-r \cdot), \Omega(\cdot)\rangle h(r) r^{-1-\alpha} d r
$$

where $f \in \mathscr{S}\left(\mathbb{R}^{n}\right), h, \Omega$ satisfy (ii) and (iii) in Theorem 1.1, respectively, and $\Omega \in H^{r}(\mathcal{M})$ satisfies

$$
\left\langle\Omega,\left.P_{m}\right|_{\mu}\right\rangle=0
$$

for all polynomials on $\mathbb{R}^{n}$ with degree $m \leq[\alpha]$ and $r=m / m+\alpha$.

When $\mathcal{M}=\mathbf{S}^{n-1}$, the operator $\mathrm{SI}_{\mathbf{S}^{n-1}, \Omega, h, \alpha}$ was studied in [4]. It is not difficult to check that (1.10) is well defined and it is finite for all $x \in \mathbb{R}^{n}$.

When $\alpha=0$, the operator $\operatorname{SI}_{S^{n-1}, \Omega, h, 0}$ is exactly the operator $\mathrm{SI}_{\mathcal{M}, \Omega, h}$.

The main result of this paper is as follows.

Theorem 1.3. Let $\mathrm{SI}_{\mathcal{M}, \Omega, h, \alpha}$ be given as in (1.10), and let $h, \mathcal{M}$ satisfy (ii) and (iii) as in Theorem 1.1, respectively. If $\Omega \in H^{r}(M)$ satisfies (1.11), then $\mathrm{SI}_{\mathcal{M}, \Omega, h, \alpha}$ extends to a bounded operator from the homogeneous Sobolev space $\dot{L}_{\alpha}^{p}\left(\mathbb{R}^{n}\right)$ to the Lebesgue space $L^{p}\left(\mathbb{R}^{n}\right)$ for $1<p<\infty$.

\section{Definitions and lemmas}

Let $M$ be a compact, smooth, $m$-dimensional manifold in $\mathbb{R}^{n}, m \leq n-1$. The Hardy spaces $H^{p}(M)$ can be defined by using the maximal operator

$$
\mathscr{A}: f \longrightarrow(\mathscr{A} f)(x)=\sup _{t>0}|u(t, x)|
$$

where $u(t, x)$ is the solution of the boundary value problem

$$
\begin{aligned}
\left(\frac{\partial}{\partial t}-\Delta_{x}\right) u & =0, \quad(t, x) \\
u(0, x) & =f(x), \quad x \in \mathbb{R}^{+} \times M,
\end{aligned}
$$

Here $\Delta_{x}$ denotes the Laplace-Beltrami operator of $\mathcal{M}$.

Definition 2.1. Define

$$
H^{p}(\mathcal{M})=\left\{f \in \mathscr{S}^{\prime}(\mathcal{M}):\|\mathscr{A} f\|_{L^{p}(\mathcal{M})}<\infty\right\} .
$$

For $f \in H^{p}(\mathcal{M})$, we set $\|f\|_{H^{p}(\mathcal{M})}=\|\mathscr{A} f\|_{L^{p}(\mathcal{M})}$.

It is well known that since $\mathcal{M}$ is compact,

$$
H^{p}(\mathcal{M})=L^{p}(\mathcal{M}) \subset L \log ^{+} L(\mathcal{M}) \subset H^{1}(\mathcal{M}) \subset H^{r}(\mathcal{M}), \quad 0<r<1<p,
$$

and all the inclusions are proper. 
Let $B_{n}(x, r)=\left\{y \in \mathbb{R}^{n}:|y-x|<r\right\}$. To give the atomic characterization of $H^{r}$, we need to define atoms on $M$.

Definition 2.2. A function $a(\cdot)$ on $\mathcal{M}$ is called an $H^{r}$ atom if there are $\rho>0$ and $\theta_{0} \in \mathcal{M}$ such that

(1) $\operatorname{supp}(a) \subseteq B_{n}\left(\theta_{0}, \rho\right) \cap \mathcal{M}$,

(2) $\|a\|_{\infty} \leq \rho^{-m / r}$,

(3) $\left.\int_{\mathcal{M}} a(\theta) P_{k}\right|_{\mu}(\theta) d \sigma(\theta)=0$,

for all polynomials $P_{k}$ on $\mathbb{R}^{n}$, with degrees $k \leq[m(1 / r-1)]$.

If $\Omega \in H^{r}(M)$, then there exist $H^{r}$ atoms $\left\{a_{j}\right\}$ and complex numbers $\left\{c_{j}\right\}$ such that

$$
\Omega=\sum c_{j} a_{j}, \quad \sum\left|c_{j}\right|^{r} \cong\|\Omega\|_{H^{r}(\mathcal{M})}^{r} \quad(\text { see }[6]) .
$$

Definition 2.3. A smooth mapping $\phi$ from an open set $U$ in $\mathbb{R}^{m}$ into $\mathbb{R}^{n}$ is said to be of finite type at $u_{0} \in U$ if, for every $\eta \in \mathbf{S}^{n-1}$, there exists a nonzero multi-index $\omega=\omega(\eta)$ such that

$$
\left.\frac{\partial^{\omega}[\eta \cdot \phi(u)]}{\partial u^{\omega}}\right|_{u=u_{0}} \neq 0 .
$$

By the smoothness and compactness of $\mathcal{M}$, we may assume that there is a smooth mapping $\phi$ from a neighborhood of $\overline{B_{m}(0,1)}$ into $\mathbb{R}^{n}$ such that

(i) $\theta_{0} \in \phi\left(B_{m}(0,1 / 2)\right)$ and $\mathcal{M} \cap B_{n}\left(\theta_{0}, \rho\right) \subset \phi\left(B_{m}(0,1)\right) \subset \mathcal{M}$;

(ii) the vectors $\partial \phi / \partial u_{1}, \ldots, \partial \phi / \partial u_{m}$ are linearly independent for each $u \in \overline{B_{m}(0,1)}$;

(iii) $\phi$ is of finite type at every point in $\overline{B_{m}(0,1)}$ (see [16, page 350]).

Thus there is a smooth function $J(u)$ such that

$$
\int_{\phi\left(B_{m}(0,1)\right)} F d \sigma=\int_{B_{m}(0,1)} F(\phi(u)) J(u) d u
$$

for any integrable function $F$ on $\mathcal{M}$. Since $\mathcal{M}$ is compact, we may assume that all $\phi$ raised from atoms $a$ satisfy $\left|\phi(u)-\phi\left(u_{0}\right)\right| \leq\left|u-u_{0}\right|$.

Now given $\Omega \in H^{r}(\mathcal{M})$, then for each $H^{r}$ atom, $a(\theta)$ supported in $M \cap B_{m}\left(\theta_{0}, \rho\right)$, write $b(u)=a(\phi(u)) J(u) \chi_{B_{m}(0,1)}$. Let $u_{0}=\phi^{-1}\left(\theta_{0}\right)$. It follows from (i)-(iii) that

$$
\begin{gathered}
\operatorname{supp}(b) \subset B_{m}\left(u_{0}, \rho\right), \\
\|b\|_{\infty} \leq C \rho^{-m / r}, \quad \text { we may assume that } C=1, \\
\int_{\mathbb{R}^{m}} b(u)\left(\phi(u)-\phi\left(u_{0}\right)\right)^{k} d u=0,
\end{gathered}
$$

for all $|k| \leq[\alpha]$, where $k=\left(k_{1}, k_{2}, \ldots, k_{m}\right)$ is a multi-index and $k=\sum_{i=1}^{m} k_{i}$.

We will need the following result (see [8]). 
LeMma 2.4. Let $\left\{a_{k}\right\}$ be a lacunary sequence of positive numbers such that $a_{k}>0$ and $\inf _{k \in \mathbb{Z}}\left|a_{k+1} / a_{k}\right|=\tau>1$. Let $\tau_{k}$ be a sequence of Borel measures in $\mathbb{R}^{n}$. Suppose that $\left\|\tau_{k}\right\| \leq 1$ and

(1) $\left|\hat{\tau}_{k}\right| \leq C\left|a_{k+1} \xi\right|^{\gamma}$,

(2) $\left|\hat{\tau}_{k}\right| \leq C\left|a_{k} \xi\right|^{-\gamma}$,

for all $k \in \mathbb{Z}$, and suppose also that for some $q>1$,

(3) $\left\|\tau^{*}(f)\right\| \leq C\|f\|_{q}$,

where $\tau^{*}$ is the maximal operator: $\tau^{*}(f)=\sup _{k}||\left|\tau_{k}\right| * f \mid$. Then

$$
T f(x)=\sum_{k=-\infty}^{\infty} \tau_{k} * f(x)
$$

is a bounded operator on $L^{p}\left(\mathbb{R}^{n}\right)$ for $|1 / p-1 / 2|<1 / 2 q$.

We will also need the following result (see $[8,9,11])$.

Lemma 2.5. Let $l, n \in \mathbb{N}$, and $\left\{\tau_{s, k}: 0 \leq s \leq l\right.$, and $\left.k \in \mathbb{Z}\right\}$ be a family of measures on $\mathbb{R}^{n}$ with $\tau_{0, k}=0$ for every $k \in \mathbb{Z}$. Let $\left\{\alpha_{s j}: 1 \leq s \leq l\right.$, and $\left.j=1,2\right\} \subset \mathbb{R}^{+},\left\{\eta_{s}: 1 \leq s \leq l\right\} \subset$ $\mathbb{R}^{+} \backslash\{1\},\left\{M_{s}: 1 \leq s \leq l\right\} \subset \mathbb{N}$, and $L_{s}: \mathbb{R}^{n} \rightarrow \mathbb{R}^{m}$ be linear transformations for $1 \leq s \leq l$. Suppose that

(i) $\left\|\tau_{s, k}\right\| \leq 1$ for $k \in \mathbb{Z}$ and $0 \leq s \leq l$;

(ii) $\left\|\hat{\tau}_{s, k}(\xi)\right\| \leq C\left(\eta_{s}^{k}\left|L_{s} \xi\right|\right)^{-\alpha_{s 2}}$ for $\xi \in \mathbb{R}^{m}, k \in \mathbb{Z}$, and $0 \leq s \leq l$;

(iii) $\left\|\hat{\tau}_{s, k}(\xi)-\hat{\tau}_{s-1, k}(\xi)\right\| \leq C\left(\eta_{s}^{k}\left|L_{s} \xi\right|\right)^{\alpha_{s 1}}$ for $\xi \in \mathbb{R}^{m}, k \in \mathbb{Z}$, and $0 \leq s \leq l$;

(iv) for some $\rho_{0}>2$, there exists a $C>0$ such that

$$
\left\|\sum_{k \in \mathbb{Z}}\left(\left|\tau_{s, k} * g_{k}\right|^{2}\right)^{1 / 2}\right\|_{L^{p_{0}\left(\mathbb{R}^{n}\right)}} \leq C\left\|\sum_{k \in \mathbb{Z}}\left(\left|g_{k}\right|^{2}\right)^{1 / 2}\right\|_{L^{p_{0}\left(\mathbb{R}^{n}\right)}},
$$

for all $\left\{g_{k}\right\} \in L^{p_{0}}\left(\mathbb{R}^{n}, l^{2}\right)$ and $1 \leq s \leq l$.

Then for every $p \in\left(p_{0}^{\prime}, p_{0}\right)$, there exists a positive constant $C_{p}$ such that

$$
\begin{gathered}
\left\|\sum_{k \in \mathbb{Z}} \tau_{l, k} * f\right\|_{L^{p}\left(\mathbb{R}^{n}\right)} \leq C_{p}\|f\|_{L^{p}\left(\mathbb{R}^{n}\right)}, \\
\left\|\left(\sum_{k \in \mathbb{Z}}\left|\tau_{l, k} * f\right|^{2}\right)^{1 / 2}\right\|_{L^{p}\left(\mathbb{R}^{n}\right)} \leq C_{p}\|f\|_{L^{p}\left(\mathbb{R}^{n}\right)}
\end{gathered}
$$

hold for all $f \in L^{p}\left(\mathbb{R}^{n}\right)$. The constant $C_{p}$ is independent of the linear transformations $\left\{L_{s}\right\}_{s=1}^{l}$.

\section{Proof of theorem}

We will prove the theorem in three different cases: $0<\alpha<1, \alpha=1,2,3, \ldots$, and $\alpha>1$, $\alpha \notin \mathbb{Z}$. Without loss of generality, we may assume that $\Omega(\theta)=a(\theta)$ is an $H^{r}$ atom as defined in Definition 2.2, the details can be found in [4]. 
Case $1(0<\alpha<1)$. Using the "lift" property of the Riesz potential and the definition of the space $\dot{L}_{\alpha}^{p}\left(\mathbb{R}^{n}\right)$, it is known that for any $\alpha>0$ and $f \in \dot{L}_{\alpha}^{p}\left(\mathbb{R}^{n}\right)$, one can write $f=G_{\alpha} * f_{\alpha}$ with $\left|\hat{G}_{\alpha}(\xi)\right| \approx|\xi|^{-\alpha},\left|G_{\alpha}(y)\right| \approx|y|^{-n+\alpha}$, and $\left\|f_{\alpha}\right\|_{p} \approx\|f\|_{\dot{L}_{\alpha}^{p}}$.

We write

$$
\left(\mathrm{SI}_{\mathcal{M}, \Omega, h, \alpha} f\right)(x)=\sum_{k} \mu_{k, \alpha} * f_{\alpha}(x)
$$

where

$$
\mu_{k, \alpha}(x)=\int_{2^{k}}^{2^{k+1}} \int_{\mathcal{M}} G_{\alpha}(x-r \theta) \Omega(\theta) h(r) r^{-1-\alpha} d \sigma(\theta) d r .
$$

In light of Lemma 2.4, in order to show that $\left\|\mathrm{SI}_{\mathcal{M}, \Omega, \alpha} f\right\|_{L^{p}} \leq C\|f\|_{L_{\alpha}^{p}}$, it suffices to show that

(i) $\left\|\mu_{k, \alpha}\right\|_{L^{1}\left(\mathbb{R}^{n}\right)} \leq C$,

(ii) $\left|\widehat{\mu}_{k, \alpha}(\xi)\right| \leq C\left|2^{k} \xi \rho\right|^{1-\alpha}$,

(iii) $\left|\hat{\mu}_{k, \alpha}(\xi)\right| \leq C\left|2^{k} \xi \rho\right|^{-\alpha}$,

(iv) $\left\|\sup _{k \in \mathbb{Z}}\left|\mu_{k, \alpha} * f\right|\right\|_{L^{q\left(\mathbb{R}^{n}\right)}} \leq C|f|_{L^{q\left(\mathbb{R}^{n}\right)}}$, for all $q \in(1, \infty)$.

Now, by the cancellation condition of $b(u)=\Omega(\phi(u)) J(u) \chi_{B_{m}(0,1)}(u)$, we have

$$
\begin{aligned}
\left\|\mu_{k, \alpha}\right\|_{L^{1}\left(\mathbb{R}^{n}\right)}= & \int_{\mathbb{R}^{n}} \mid \int_{2^{k}}^{2^{k+1}}\left[\int _ { B _ { m } ( 0 , 1 ) } \left(G_{\alpha}(x-r \phi(u))\right.\right. \\
& \left.\left.-G_{\alpha}\left(x-r \phi\left(u_{0}\right)\right)\right) b(u) d u\right]|h(r)| r^{-1-\alpha} d r \mid d x \\
\leq & \int_{2^{k}}^{2^{k+1}} r^{-1-\alpha} \int_{B_{m}(0,1)}|b(u)| \\
& \times \int_{\mathbb{R}^{n}}\left|G_{\alpha}(x-r \phi(u))-G_{\alpha}\left(x-r \phi\left(u_{0}\right)\right)\right| d x|h(r)| d u d r .
\end{aligned}
$$

Letting $y=x-r \phi\left(u_{0}\right)$, we have

$$
\int_{\mathbb{R}^{n}}\left|G_{\alpha}(x-r \phi(u))-G_{\alpha}\left(x-r \phi\left(u_{0}\right)\right)\right| d x=\int_{\mathbb{R}^{n}}\left|G_{\alpha}\left(y+r\left(\phi(u)-\phi\left(u_{0}\right)\right)\right)-G_{\alpha}(y)\right| d y .
$$

As we mentioned before, $\left|\phi(u)-\phi\left(u_{0}\right)\right| \leq\left|u-u_{0}\right| \leq \rho$, for $u \in \operatorname{supp}(b)$.

We write

$$
\begin{aligned}
\int_{\mathbb{R}^{n}} \mid & G_{\alpha}\left(y+r\left(\phi(u)-\phi\left(u_{0}\right)\right)\right)-G_{\alpha}(y) \mid d y \\
= & \int_{|y| \geq 3 r \rho}\left|G_{\alpha}\left(y+r\left(\phi(u)-\phi\left(u_{0}\right)\right)\right)-G_{\alpha}(y)\right| d y \\
& \quad+\int_{|y|<3 r \rho}\left|G_{\alpha}\left(y+r\left(\phi(u)-\phi\left(u_{0}\right)\right)\right)-G_{\alpha}(y)\right| d y \\
= & I_{1}+I_{2}, \quad \text { where } u \text { is in the support of } b(u) .
\end{aligned}
$$


8 Singular integrals along manifolds

By the definition of $G_{\alpha}(x)$, we have, if $y \geq 3 r \rho \geq 3 r\left|\phi(u)-\phi\left(u_{0}\right)\right|$,

$$
\left|G_{\alpha}\left(y+r\left(\phi(u)-\phi\left(u_{0}\right)\right)\right)-G_{\alpha}(y)\right| \leq C \frac{r \rho}{|y|^{n-\alpha+1}} .
$$

Thus,

$$
I_{1} \leq C \int_{|y| \geq 3 r \rho} \frac{r \rho}{|y|^{n-\alpha+1}} d y \approx(r \rho)^{\alpha} .
$$

It is easy to see that

$$
I_{2} \leq 2 \int_{|y| \leq 5 r \rho}\left|G_{\alpha}(y)\right| d y \leq C \int_{|y| \leq 5 r \rho} \frac{d y}{|y|^{n-\alpha}} \leq C(r \rho)^{\alpha} .
$$

Thus,

$$
\begin{aligned}
\left\|\mu_{k, \alpha}\right\|_{L^{1}\left(\mathbb{R}^{n}\right)} \leq & \int_{2^{k}}^{2^{k+1}} r^{-1-\alpha} \int_{B_{m}(0,1)}|b(u)| \\
& \times \int_{\mathbb{R}^{n}}\left|G_{\alpha}(x-r \phi(u))-G_{\alpha}\left(x-r \phi\left(u_{0}\right)\right)\right| d x|h(r)| d u d r \\
\leq & \int_{2^{k}}^{2^{k+1}} r^{-1-\alpha} \int_{B_{m}(0,1)}|b(u)|(r \rho)^{\alpha}|h(r)| d u d r \leq C .
\end{aligned}
$$

To prove (ii), we write

$$
\left.\left|\hat{\mu}_{k, \alpha}(\xi)\right|=\mid \widehat{\left(\sigma_{k, \alpha} * G_{\alpha}\right.}\right)\left.(\xi)|=| \hat{\sigma}_{k, \alpha}(\xi)|| \widehat{G}_{\alpha}(\xi)|\leq C| \xi\right|^{-\alpha}\left|\hat{\sigma}_{k, \alpha}(\xi)\right| .
$$

Thus,

$$
\begin{aligned}
\left|\hat{\mu}_{k, \alpha}(\xi)\right| & \leq C|\xi|^{-\alpha}\left|\int_{2^{k}}^{2^{k+1}}\left(\int_{B_{m}(0,1)} e^{-i r \xi \cdot \phi(u)} b(u) d u\right) r^{-1-\alpha} h(r) d r\right| \\
& \leq C|\xi|^{-\alpha} 2^{-k \alpha} \int_{2^{k}}^{2^{k+1}}\left|\int_{B_{m}(0,1)}\left(e^{-i r \xi \cdot \phi(u)}-e^{i r \xi \cdot \phi\left(u_{0}\right)}\right) b(u) d u\right| r^{-1}|h(r)| d r \\
& \leq C|\xi|^{-\alpha} 2^{-k \alpha}\left|2^{k} \xi\right| \int_{B_{m}(0,1)}\left|\phi(u)-\phi\left(u_{0}\right)\right||b(u)| d u \leq C\left|2^{k} \xi \rho\right|^{1-\alpha},
\end{aligned}
$$

which proves (ii).

On the other hand,

$$
\left|\widehat{\mu}_{k, \alpha}(\xi)\right| \leq C|\xi|^{-\alpha} 2^{-k \alpha} \int_{2^{k}}^{2^{k+1}} \int_{B_{m}(0,1)}|b(u)| d u r^{-1}|h(r)| d r=C\left|2^{k} \xi \rho\right|^{-\alpha}
$$

which proves (iii). 
It remains to show that

$$
\left\|\sup _{k \in \mathbb{Z}}\left|\mu_{k, \alpha}\right| * f\right\|_{p} \leq C\|f\|_{p}
$$

Without loss of generality, assume that $h(r) \geq 0$. Then

$$
\begin{aligned}
\left\|\sup _{k \in \mathbb{Z}}\left|\mu_{k, \alpha}\right| * f\right\|_{L^{q\left(\mathbb{R}^{n}\right)}} & \\
\leq \underset{k \in \mathbb{Z}}{\leq} 2^{-k-k \alpha} \int_{2^{k}}^{2^{k+1}} h(r) \int_{B_{m}(0,1)}|b(u)| \int_{\mathbb{R}^{n}}|f(x-z)| \mid G_{\alpha}(z-r \phi(u)) & -G_{\alpha}\left(z-r \phi\left(u_{0}\right)\right) \mid d z d u d r .
\end{aligned}
$$

In the above integral, we write

$$
\begin{aligned}
\int_{\mathbb{R}^{n}}|f(x-z)|\left|G_{\alpha}(z-r \phi(u))-G_{\alpha}\left(z-r \phi\left(u_{0}\right)\right)\right| d z \\
=\int_{\left|z-r \phi\left(u_{0}\right)\right|>3 r \rho}|f(x-z)|\left|G_{\alpha}(z-r \phi(u))-G_{\alpha}\left(z-r \phi\left(u_{0}\right)\right)\right| d z \\
\quad+\int_{\left|z-r \phi\left(u_{0}\right)\right| \leq 3 r \rho}|f(x-z)|\left|G_{\alpha}(z-r \phi(u))-G_{\alpha}\left(z-r \phi\left(u_{0}\right)\right)\right| d z \\
=I_{1}(f)(x)+I_{2}(f)(x),
\end{aligned}
$$

where $u \in B_{n}\left(u_{0}, \rho\right) \cap M$.

In the integral $I_{1}(f)$, we change variables $z-r \phi\left(u_{0}\right) \rightarrow y$ and again write $y$ as $z$, then

$$
I_{1}(f)(x)=C \int_{|z|>3 r \rho}\left|f\left(x-z+r \phi\left(u_{0}\right)\right)\right|\left|G_{\alpha}\left(z+r \phi\left(u_{0}\right)-r \phi(u)\right)-G_{\alpha}(z)\right| d z .
$$

Note that $\left|r \phi\left(u_{0}\right)-r \phi(u)\right| \leq r \rho<|z| / 2$. By the mean value theorem,

$$
\begin{aligned}
I_{1}(f)(x) & \leq C \int_{|z|>3 r \rho} r \rho\left|f\left(x-z+r \phi\left(u_{0}\right)\right)\right||z|^{\alpha-1-n} d z \\
& \cong \int_{S^{n-1}} \int_{3 r \rho}^{\infty} r \rho s^{\alpha-2}\left|f\left(x-s z^{\prime}+r \phi\left(u_{0}\right)\right)\right| d s d \sigma\left(z^{\prime}\right) .
\end{aligned}
$$

Using integration by parts, it is easy to see that

$$
\begin{aligned}
I_{1}(f)(x) \leq & C \int_{S^{n-1}}(r \rho)^{\alpha}(r \rho)^{-1} \int_{0}^{3 r \rho}\left|f\left(x-t z^{\prime}+r \phi\left(u_{0}\right)\right)\right| d t d \sigma\left(z^{\prime}\right) \\
& +C \int_{S^{n-1}} \int_{3 r \rho}^{\infty} r \rho s^{\alpha-3} \int_{0}^{s}\left|f\left(x-t z^{\prime}+r \phi\left(u_{0}\right)\right)\right| d s d t d \sigma\left(z^{\prime}\right) .
\end{aligned}
$$


10 Singular integrals along manifolds

Let $M_{z} f(x)$ be the maximal function

$$
M_{z} f(x)=\sup _{t>0} t^{-1} \int_{0}^{t} \mid f(x-r z) d r .
$$

It is known in [16, page 477] that there is a constant $C$ independent of $z$ such that

$$
\left\|M_{z}(f)\right\|_{L^{p\left(\mathbb{R}^{n}\right)}} \leq C\|f\|_{L^{p}\left(\mathbb{R}^{n}\right)}
$$

Thus we have

$$
I_{1}(f)(x) \leq C(r \rho)^{\alpha} \int_{S^{n-1}} M_{z^{\prime}} f\left(x+r \phi\left(u_{0}\right)\right) d \sigma\left(z^{\prime}\right) .
$$

For the second integral $I_{2}(f)(x)$, we have $I_{2}(f)(x) \leq J_{1}(f)(x)+J_{2}(f)(x)$, where

$$
\begin{aligned}
& J_{1}(f)(x)=\int_{\left|z-r \phi\left(u_{0}\right)\right|<3 r \rho}\left|f(x-z) G_{\alpha}(z-r \phi(u))\right| d z, \\
& J_{2}(f)(x)=\int_{|z|<3 r \rho}\left|f\left(x-z+r \phi\left(u_{0}\right)\right) G_{\alpha}(z)\right| d z .
\end{aligned}
$$

Let $w=z-r \phi(u)$. Then, in $J_{1}(f)(x)$, we have

$$
|w| \leq\left|z-r \phi\left(u_{0}\right)\right|+\left|r \phi(u)-r \phi\left(u_{0}\right)\right| \leq 4 r \rho .
$$

This gives (again write $z$ instead of $w$ )

$$
\begin{aligned}
J_{1}(f)(x) & \leq\left. C \int_{|z|<4 r \rho}|f(x-z-r \phi(u))| z\right|^{\alpha-n} d z \\
& =C \int_{S^{n-1}} \int_{0}^{4 r \rho} t^{\alpha-1} \mid f\left(x-t z^{\prime}-r \phi(u)\right) d t d \sigma\left(z^{\prime}\right) .
\end{aligned}
$$

Using integration by parts, we obtain

$$
J_{1}(f)(x) \leq C \int_{S^{n-1}}(r \rho)^{\alpha} M_{z^{\prime}}(f(x-r \phi(u))) d \sigma\left(z^{\prime}\right) .
$$

Similarly, we can have the same estimate on $J_{2}(f)(x)$ so that

$$
J_{2}(f)(x) \leq C \int_{S^{n-1}}(r \rho)^{\alpha}\left\{M_{z^{\prime}} f\left(x+r \phi\left(u_{0}\right)\right)+M_{z^{\prime}}(f(x-r \phi(u)))\right\} d \sigma\left(z^{\prime}\right) .
$$

Thus

$$
\begin{aligned}
& \int_{\mathbb{R}^{n}}|f(x-z)|\left|G_{\alpha}(z-r \phi(u))-G_{\alpha}\left(z-r \phi\left(u_{0}\right)\right)\right| d z \\
& \quad \leq C(r \rho)^{\alpha} \int_{S^{n-1}}\left\{M_{z^{\prime}} f\left(x+r \phi\left(u_{0}\right)\right)+M_{z^{\prime}}(f(x-r \phi(u)))\right\} d \sigma\left(z^{\prime}\right) .
\end{aligned}
$$


Therefore, we have

$$
\begin{aligned}
& \left\|\sup _{k \in \mathbb{Z}}\left|\mu_{k, \alpha}\right| * f\right\|_{L^{q}\left(\mathbb{R}^{n}\right)} \\
& \quad \leq C \int_{B_{m}(0,1) \times \mathbf{S}^{n-1}}|b(u)| \rho^{\alpha}\left\{\left\|M_{\phi\left(u_{0}\right)} M_{z^{\prime}}(f)\right\|_{L^{q}\left(\mathbb{R}^{n}\right)}+\left\|M_{\phi(u)} M_{z^{\prime}} f\right\|_{L^{q}\left(\mathbb{R}^{n}\right)}\right\} d \sigma\left(z^{\prime}\right) d u .
\end{aligned}
$$

Since $b$ is an $(r, \infty)$ atom supported in $B_{m}\left(u_{0}, \rho\right) \cap \mathcal{M}$ with $r=m /(m+\alpha)$, it is easy to see that

$$
\int_{B_{m}(0,1)}|b(u)| \rho^{\alpha} d u \leq C
$$

uniformly for $b$ and $\rho$. Thus

$$
\left\|\sup _{k \in \mathbb{Z}}\left|\mu_{k, \alpha}\right| * f\right\|_{L^{q\left(\mathbb{R}^{n}\right)}} \leq C\|f\|_{L^{q\left(\mathbb{R}^{n}\right)} .}
$$

By Lemma 2.4, Case 1 is established.

Case $2(\alpha=1,2,3, \ldots)$. Using Taylor's expansion about $\theta_{0}$, we have, for $j=\left(j_{1}, \ldots, j_{m}\right)$,

$$
\begin{aligned}
\left(\mathrm{SI}_{\mathcal{M}, \Omega, h, \alpha} f\right)(x)= & \sum_{|j|=\alpha} C_{j} \int_{0}^{1}(1-t)^{\alpha-1} \int_{0}^{\infty} \int_{B_{m}(0,1)} \mathscr{B}(u) r^{-1} h(r) \\
& \times D^{j} f\left(x-r \phi\left(u_{0}\right)+r t\left(\phi\left(u_{0}\right)-\phi(u)\right)\right) d u d r d t
\end{aligned}
$$

where $C_{j}$ 's are constants and $\mathscr{B}(u)=b(u)\left(\phi(u)-\phi\left(u_{0}\right)\right)^{j}$. Clearly, $\mathscr{B}(u)$ is an $H^{1}$ atom with the same support as $b$.

For each $j,|j|=\alpha$, define the measures $\left\{\sigma_{\phi, \Re, h, k, \alpha} \mid k \in \mathbb{Z}\right\}$ on $\mathbb{R}^{n}$ by

$$
\begin{aligned}
\int_{\mathbb{R}^{n}} F(x) d \sigma_{\phi, \Re, h, k, \alpha} \\
\quad=\int_{0}^{1}(1-t)^{\alpha-1} \int_{2^{k}}^{2^{k+1}} \int_{B_{m}(0,1)} F\left(x-r \phi\left(u_{0}\right)+r t\left(\phi\left(u_{0}\right)-\phi(u)\right)\right) \mathscr{B}(u) r^{-1} h(r) d u d r d t .
\end{aligned}
$$

Lemma 3.1. Suppose that $h$ satisfies (ii) in Theorem 1.1. Then for $1<p<\infty$, there exists a constant $C_{p}>0$ such that

$$
\left\|\left(\sum_{k \in \mathbb{Z}}\left|\sigma_{\phi, \mathscr{B}, h, k, \alpha} * g_{k}\right|^{2}\right)^{1 / 2}\right\|_{p} \leq C_{p}\left\|\left(\sum_{k \in \mathbb{Z}}\left|g_{k}\right|^{2}\right)^{1 / 2}\right\|_{p}
$$

holds for all continuous mappings $\phi$ and measurable functions $\left\{g_{k}\right\}$ on $\mathbb{R}^{n}$. 
12 Singular integrals along manifolds

Proof. For $\xi \in \mathbb{R}^{n}$, we define the maximal operator $M_{\xi}$ on $\mathbb{R}^{n}$ by

$$
\left(M_{\xi} f\right)(x)=\sup _{k \in \mathbb{Z}}\left[2^{-k} \int_{2^{k}}^{2^{k+1}}|f(x+r \xi)| d r\right] .
$$

It follows from the $L^{p}$-boundedness of the one-dimensional Hardy-Littlewood maximal operator that

$$
\left\|M_{\xi} f\right\|_{p} \leq A_{p}\|f\|_{p}
$$

for $1<p \leq \infty$, where $A_{p}$ is independent of $\xi$.

By duality, we may assume that $p>2$, then for $\left\{g_{k}\right\} \in L^{p}\left(\mathbb{R}^{n}, l^{2}\right)$, there exists a function $w \in L^{(p / 2)^{\prime}}\left(\mathbb{R}^{n}\right)$ such that $\|w\|_{(p / 2)^{\prime}}=1$ and

$$
\left\|\left(\sum_{k \in \mathbb{Z}}\left|\sigma_{\phi, \Re, h, k, \alpha} * g_{k}\right|^{2}\right)^{1 / 2}\right\|_{p}^{2}=\int_{\mathbb{R}^{n}}\left(\sum_{k \in \mathbb{Z}}\left|\sigma_{\phi, \Re, h, k, \alpha} * g_{k}\right|^{2}\right) w(x) d x .
$$

By Hölder's inequality and (3.35),

$$
\begin{aligned}
& \left\|\left(\sum_{k \in \mathbb{Z}}\left|\sigma_{\phi, \Re, h, k, \alpha} * g_{k}\right|^{2}\right)^{1 / 2}\right\|_{p}^{2} \\
& \leq \int_{\mathbb{R}^{n}} \sum_{k \in \mathbb{Z}} \mid \int_{0}^{1}(1-t)^{\alpha-1} \int_{2^{k}}^{2^{k+1}} \int_{B_{m}(0,1)} g_{k}\left(x r \phi\left(u_{0}\right)+r t\left(\phi\left(u_{0}\right)-\phi(u)\right)\right) \\
& \times\left.\mathscr{B}(u) r^{-1} h(r) d u d r d t\right|^{2} w(x) d x \\
& \leq C\|\mathscr{B}\|_{1} \sum_{k \in \mathbb{Z}} 2^{-k} \int_{\mathbb{R}^{n}} \int_{0}^{1} \int_{2^{k}}^{2^{k+1}} \int_{B_{m}(0,1)}\left|g_{k}\left(x-r \phi\left(u_{0}\right)+r t\left(\phi\left(u_{0}\right)-\phi(u)\right)\right)\right|^{2} \\
& \times|\mathscr{B}(u) w(x)| d u d r d t d x \\
& =C\|\mathscr{B}\|_{1} \int_{0}^{1} \int_{B_{m}(0,1)}|\mathscr{B}(u)| \\
& \times\left[\sum_{k \in \mathbb{Z}} 2^{-k} \int_{2^{k}}^{2^{k+1}} \int_{\mathbb{R}^{n}}\left|g_{k}(x)\right|^{2}\left|w\left(x+r \phi\left(u_{0}\right)+r t\left(\phi\left(u_{0}\right)-\phi(u)\right)\right)\right| d x d r\right] d u d t \\
& \leq C\|\mathscr{B}\|_{1} \int_{0}^{1} \int_{B_{m}(0,1)}\left[\int_{\mathbb{R}^{n}}\left(\sum_{k \in \mathbb{Z}}\left|g_{k}(x)\right|^{2}\right)\left(M_{\phi\left(u_{0}\right)+t\left(\phi\left(u_{0}\right)-\phi(u)\right)} w\right)(x) d x\right]|\mathscr{B}(u)| d u d t \\
& \leq C\|\mathscr{B}\|_{1}^{2}\left\|\left(\sum_{k \in \mathbb{Z}}\left|g_{k}\right|^{2}\right)^{1 / 2}\right\|_{p}^{2} \text {. }
\end{aligned}
$$

We also have the following estimates for $\sigma_{\phi, \Re, h, k, \alpha}$. 
LemmA 3.2. Suppose that $\phi$ is smooth and of finite type at every point in $\overline{B_{m}(0,1)}$ and $h$ satisfies (ii) in Theorem 1.1. Then there exists a $\delta>0$ such that

$$
\left|\hat{\sigma}_{\phi, \Re, h, k, \alpha}\right| \leq C\|\mathscr{S}\|_{2}\left(2^{k}|\xi|\right)^{-\delta}
$$

Proof.

$$
\left|\hat{\sigma}_{\phi, \Re, h, k, \alpha}(\xi)\right|=\left|\int_{0}^{1}(1-t)^{\alpha-1} \int_{2^{k}}^{2^{k+1}} h(r) r^{-1} e^{i \xi r \phi\left(u_{0}\right)} e^{-i \xi r t \phi\left(u_{0}\right)} \int_{B_{m}(0,1)} \mathscr{B}(u) e^{i \xi r t \phi(u)} d u d r d t\right| .
$$

Changing variables $(s=r t)$, we have

$$
\begin{aligned}
\left|\hat{\sigma}_{\phi, \mathscr{B}, h, k, \alpha}(\xi)\right|= & \mid \int_{0}^{1}(1-t)^{\alpha-1} \int_{2^{k} t}^{2^{k+1} t} h\left(\frac{s}{t}\right) s^{-1} e^{i \xi(s / t) \phi\left(u_{0}\right)} e^{-i \xi s \phi\left(u_{0}\right)} \\
& \times \int_{B_{m}(0,1)} \mathscr{B}(u) e^{i \xi s \phi(u)} d u d s d t \mid \\
\leq & \int_{0}^{1}\left|(1-t)^{\alpha-1}\right| \int_{2^{k} t}^{2^{k+1} t}\left|h(s / t) s^{-1}\right|\left|\left(\int_{B_{m}(0,1)} \mathscr{B}(u) e^{i \xi s \phi(u)} d u\right)\right| d s d t .
\end{aligned}
$$

The remainder of the proof is similar to the proof of Lemma 3.3 in [5].

The following result is similar to those in [10], see also [5].

Lemma 3.3. Let $\mathscr{B}(\cdot)$ be a function satisfying $\operatorname{supp}(\mathscr{B}) \subset B_{m}(0, \rho)$ and $\|\mathscr{B}\|_{\infty} \leq \rho^{-m}$ for some $\rho<1$. Suppose that $h$ satisfies (ii) in Theorem 1.1. Then there exists a constant $C>0$ such that

$$
\begin{aligned}
& \left|\int_{0}^{1}(1-t)^{\alpha-1} \int_{2^{k}}^{2^{k+1}} h(r) r^{-1}\left(\int_{B_{m}(0,1)} \mathscr{B}(u) e^{-i r t\left[Q(u)+\sum_{|\beta|=s} d_{\beta} u^{\beta}\right]} d u\right) d r d t\right| \\
& \quad \leq C\left(2^{k} \rho^{s} \sum_{|\beta|=s}\left|d_{\beta}\right|\right)^{-1 /(4 s)}
\end{aligned}
$$

holds for all polynomials $Q: \mathbb{R}^{m} \rightarrow \mathbb{R}$ with $\operatorname{deg}(Q)<s$ and $\left\{d_{\beta}\right\} \subset \mathbb{R}$. The constant $C$ is independent of $\rho$.

Now, by Lemma 3.2, there exists a $\delta>0$ such that

$$
\left|\hat{\sigma}_{\phi, \Re, h, k, \alpha}(\xi)\right| \leq C\left(2^{k}|\xi|\right)^{-\delta} \rho^{-m / 2} .
$$


14 Singular integrals along manifolds

Let $l=[m /(2 \delta)]+1$. Following the proof of Theorem 3.7 in [5], we define a sequence of mappings $\left\{\Phi^{s}\right\}_{s=0}^{s=l}$ by

$$
\begin{gathered}
\Phi^{l}=\phi=\left(\phi_{1}, \ldots, \phi_{n}\right), \\
\Phi^{s}(u)=\left(\sum_{|\beta| \leq s} \frac{1}{\beta !} \frac{\partial^{\beta} \phi_{1}\left(u_{0}\right)}{\partial u^{\beta}}\left(u-u_{0}\right)^{\beta}, \ldots, \sum_{|\beta| \leq s} \frac{1}{\beta !} \frac{\partial^{\beta} \phi_{n}\left(u_{0}\right)}{\partial u^{\beta}}\left(u-u_{0}\right)^{\beta}\right)
\end{gathered}
$$

for $s=0,1, \ldots, l-1$.

Let

$$
\sigma_{s, k, \alpha}=\sigma_{\Phi^{s}, \Re, h, k, \alpha}
$$

for $0 \leq s \leq l$ and $k \in \mathbb{Z}$.

In order to show that $\left\|\mathrm{SI}_{\mathcal{M}, \Omega, h, \alpha} f\right\|_{L^{p}} \leq C\|f\|_{L_{\alpha}^{p}}$, it suffices to show that the family of measures $\left\{\sigma_{s, k, \alpha}\right\}$ satisfies the conditions of Lemma 2.5.

By its definition and Lemma 3.2, the family of measures $\left\{\sigma_{s, k, \alpha}\right\}$ satisfies conditions (i) and (iv) in Lemma 2.5, for any $p_{0}>2$.

It is easy to see that

$$
\left\|\sigma_{s, k, \alpha}\right\| \leq\|\Re\|_{1} \int_{0}^{1}\left|(1-t)^{\alpha-1}\right| \int_{2^{k}}^{2^{k+1}} r^{-1}|h(r)| d r d t \leq C .
$$

Also we have

$$
\sigma_{0, k, \alpha}(x)=0, \quad \text { by the cancellation condition of } \mathscr{B}(u) \text {. }
$$

For $j=1, \ldots, n$, let

$$
d_{j, \beta}=\frac{1}{\beta !} \frac{\partial^{\beta} \phi_{j}\left(u_{0}\right)}{\partial u^{\beta}}
$$

By (3.42) and Lemma 3.3, we have

$$
\begin{gathered}
\left|\widehat{\sigma}_{l, k, \alpha}(\xi)\right| \leq C\left(2^{k} \rho^{l}|\xi|\right)^{-\delta} \\
\left|\hat{\sigma}_{s, k, \alpha}(\xi)\right| \leq C\left(2^{k} \rho^{s} \sum_{|\beta|=s}\left|\sum_{j=1}^{n} d_{j \beta} \xi_{j}\right|\right)^{-1 /(4 s)}
\end{gathered}
$$

for $1 \leq s \leq l-1, k \in \mathbb{Z}$ and $\xi \in \mathbb{R}^{n}$. We also have,

$$
\begin{aligned}
& \left|\hat{\sigma}_{l, k, \alpha}(\xi)-\hat{\sigma}_{l-1, k, \alpha}(\xi)\right| \\
& \quad \leq\left|\int_{0}^{1}\right|(1-t)^{\alpha-1}\left|\int_{2^{k}}^{2^{k+1}}\right| h(r)\left|r^{-1} \int_{B_{m}(0,1)}\right| \mathscr{B}(u)|| e^{i \xi r t \phi(u)}-e^{i \xi r t \phi^{l-1}(u)}|d u d r d t| \\
& \quad \leq C|\xi| 2^{k} \int_{B_{m}(0,1)}|\mathscr{B}(u)|\left|\left(\phi(u)-\phi^{l-1}(u)\right)\right| d u \leq C\left(2^{k}|\xi| \rho^{l}\right) .
\end{aligned}
$$


Similarly,

$$
\begin{aligned}
\left|\hat{\sigma}_{s, k, \alpha}(\xi)-\hat{\sigma}_{s-1, k, \alpha}(\xi)\right| & \leq C 2^{k} \int_{B_{m}(0,1)}|\mathscr{B}(u)|\left|\xi \cdot\left(\phi^{s}(u)-\phi^{s-1}(u)\right)\right| d u \\
& \leq C 2^{k} \rho^{s} \sum_{|\beta|=s}\left|\sum_{j=1}^{n} d_{j \beta} \xi_{j}\right|
\end{aligned}
$$

for $1 \leq s \leq l-1, k \in \mathbb{Z}$ and $\xi \in \mathbb{R}^{n}$.

Invoking Lemma 2.5, Case 2 is established.

Case $3(\alpha>1, \alpha \notin \mathbb{Z})$. Write $\alpha=[\alpha]+\gamma, \gamma \in(0,1)$.

Similar to the case $\alpha=1,2,3, \ldots$, by Taylor's expansion, we have

$$
\begin{aligned}
\left(\mathrm{SI}_{\mathcal{M}, \Omega, h, \alpha} f\right)(x)= & \sum_{|j|=\alpha} C_{j} \int_{0}^{1}(1-t)^{\alpha-1} \int_{0}^{\infty} r^{-1-\gamma} h(r) \int_{B_{m}(0,1)} \mathscr{B}(u) \\
& \times D^{j} f\left(x-r \phi\left(u_{0}\right)+r t\left(\phi\left(u_{0}\right)-\phi(u)\right)\right) d u d t d r,
\end{aligned}
$$

where $\mathscr{B}(u)=b(u)\left(\phi(u)-\phi\left(u_{0}\right)\right)^{j}$. Clearly, $\mathscr{B}(u)$ is an $H^{r}$ atom, where $r=m /(m+\gamma)$.

Similar to Case 1, again using the "lift" property of the Riesz potential and the definition of the space $\dot{L}_{\alpha}^{p}\left(\mathbb{R}^{n}\right)$, it is known that for any $\gamma>0$ and $f \in \dot{L}_{\alpha}^{p}\left(\mathbb{R}^{n}\right)$, one can write $f=G_{\gamma} * f_{\gamma}$ with $\left|\hat{G}_{\gamma}(\xi)\right| \approx|\xi|^{-\gamma},\left|G_{\gamma}(y)\right| \approx|y|^{-n+\gamma}$, and $\left\|f_{\gamma}\right\|_{p} \approx\|f\|_{\dot{L}_{\gamma}^{p}}$.

We write

$$
\left(\mathrm{SI}_{\mathcal{M}, \Omega, h, \alpha, k} f\right)(x)=\sum_{k} \sigma_{k, \gamma} * f_{\gamma}
$$

where

$$
\begin{aligned}
\sigma_{k, \gamma}= & \int_{0}^{1}(1-t)^{\alpha-1} \int_{2^{k}}^{2^{k+1}} r^{-1-\gamma} h(r) \int_{B_{m}(0,1)} \mathscr{B}(u) G_{\gamma}\left(x-r \phi\left(u_{0}\right)+r t\left(\phi\left(u_{0}\right)-\phi(u)\right)\right) d u d r d t \\
= & \int_{0}^{1}(1-t)^{\alpha-1} \int_{2^{k}}^{2^{k+1}} r^{-1-\gamma} h(r) \int_{B_{m}(0,1)} \mathscr{B}(u) \\
& \times\left[G_{\gamma}\left(x-r \phi\left(u_{0}\right)+r t\left(\phi\left(u_{0}\right)-\phi(u)\right)\right)-G_{\gamma}\left(x-r \phi\left(u_{0}\right)\right)\right] d u d r d t .
\end{aligned}
$$

Again, by Lemma 2.4, in order to show that $\left\|\mathrm{SI}_{\mathcal{M}, \Omega, h, \alpha, k} f\right\|_{L^{p}} \leq C\|f\|_{\dot{L}_{\gamma}^{p}}$, it suffices to show that

(i) $\left\|\sigma_{k, \gamma}\right\|_{L^{1}\left(\mathbb{R}^{n}\right)} \leq C$,

(ii) $\left|\widehat{\sigma}_{k, \gamma}(\xi)\right| \leq C\left|2^{k} \xi \rho\right|^{1-\gamma}$,

(iii) $\left|\hat{\sigma}_{k, \gamma}(\xi)\right| \leq C\left|2^{k} \xi \rho\right|^{-\gamma}$,

(iv) $\left\|\sup _{k \in \mathbb{Z}}\left|\sigma_{k, \gamma}\right| * f\right\|_{L^{q\left(\mathbb{R}^{n}\right)}} \leq C|f|_{L^{q\left(\mathbb{R}^{n}\right)}}$.

The proof is similar to the proof for Case 1. We leave the details to the reader.

\section{Acknowledgment}

The work of the second author was partially supported by NSF in China (Grant no. 10371046). 


\section{References}

[1] A. P. Calderón and A. Zygmund, On the existence of certain singular integrals, Acta Mathematica 88 (1952), 85-139.

[2] __ On singular integrals, American Journal of Mathematics 78 (1956), no. 2, 289-309.

[3] L.-K. Chen, On a singular integral, Studia Mathematica 85 (1986), no. 1, 61-72 (1987).

[4] J. Chen, D. Fan, and Y. Ying, Certain operators with rough singular kernels, Canadian Journal of Mathematics 55 (2003), no. 3, 504-532.

[5] L. C. Cheng and Y. Pan, On an extension of Calderón-Zygmund operators, Illinois Journal of Mathematics 46 (2002), no. 4, 1079-1088.

[6] L. Colzani and G. Travaglini, Hardy-Lorentz spaces and expansions in eigenfunctions of the Laplace-Beltrami operator on compact manifolds, Colloquium Mathematicum 58 (1990), no. 2, 305-315.

[7] W. C. Connett, Singular integrals near L ${ }^{1}$, Harmonic Analysis in Euclidean Spaces (Proc. Sympos. Pure Math., Williams Coll., Williamstown, Mass, 1978), Part 1 (S. Waigner and G. Weiss, eds.), Proc. Sympos. Pure Math., vol. 35, American Mathematical Society, Rhode Island, 1979, pp. 163-165.

[8] J. Duoandikoetxea and J. L. Rubio de Francia, Maximal and singular integral operators via Fourier transform estimates, Inventiones Mathematicae 84 (1986), no. 3, 541-561.

[9] D. Fan, K. Guo, and Y. Pan, $L^{p}$ estimates for singular integrals associated to homogeneous surfaces, Journal für die reine und angewandte Mathematik 542 (2002), 1-22.

[10] D. Fan and Y. Pan, On the Fourier transform of measures carried by submanifolds of finite type, Journal d'Analyse Mathématique 71 (1997), 135-147.

[11] _ Singular integral operators with rough kernels supported by subvarieties, American Journal of Mathematics 119 (1997), no. 4, 799-839.

[12] R. Fefferman, A note on singular integrals, Proceedings of the American Mathematical Society 74 (1979), no. 2, 266-270.

[13] L. Grafakos and A. Stefanov, Convolution Calderón-Zygmund singular integral operators with rough kernels, Analysis of Divergence (Orono, ME, 1997), Applied and Numerical Harmonic Analysis, Birkhäuser Boston, Massachusetts, 1999, pp. 119-143.

[14] J. Namazi, A singular integral, Ph.D. thesis, Indiana University, Indiana, 1984.

[15] F. Ricci and G. Weiss, A characterization of $H^{1}\left(\sum_{n-1}\right)$, Harmonic Analysis in Euclidean Spaces (Proc. Sympos. Pure Math., Williams Coll., Williamstown, Mass, 1978), Part 1 (S. Waigner and G. Weiss, eds.), Proc. Sympos. Pure Math., vol. 35, American Mathematical Society, Rhode Island, 1979, pp. 289-294.

[16] E. M. Stein, Harmonic Analysis: Real-Variable Methods, Orthogonality, and Oscillatory Integrals, Princeton Mathematical Series, vol. 43, Princeton University Press, New Jersey, 1993.

Abdelnaser Al-Hasan: Department of Mathematics, An-Najah National University P.O. Box 7, Nablus, West Bank, Palestine

E-mail address: alhasana@mtmary.edu

Dashan Fan: Department of Mathematics, University of Wisconsin-Milwaukee, Milwaukee, WI 53201, USA; Huazhong Normal University of China, Wuhan 43007, China

E-mail address: fan@uwm.edu 


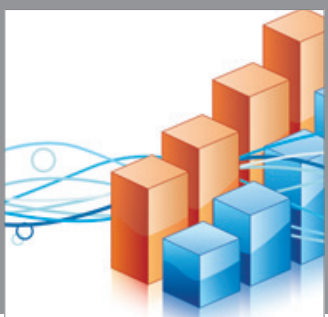

Advances in

Operations Research

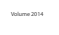

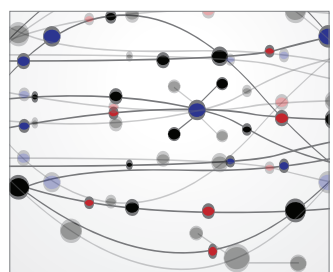

\section{The Scientific} World Journal
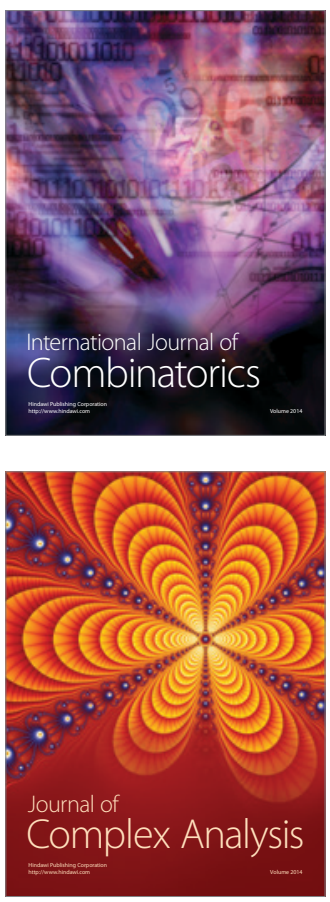

International Journal of

Mathematics and

Mathematical

Sciences
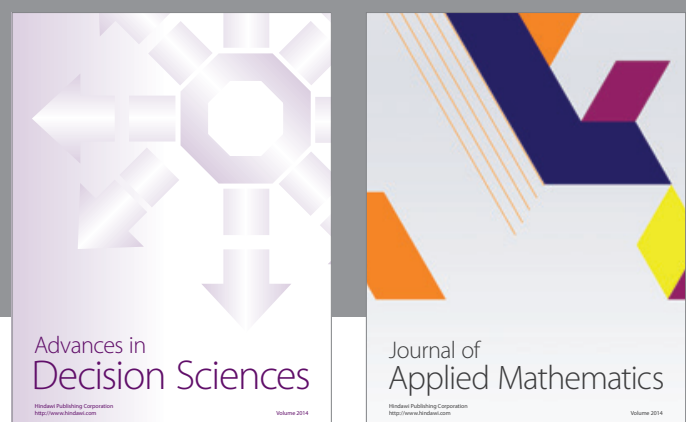

Journal of

Applied Mathematics
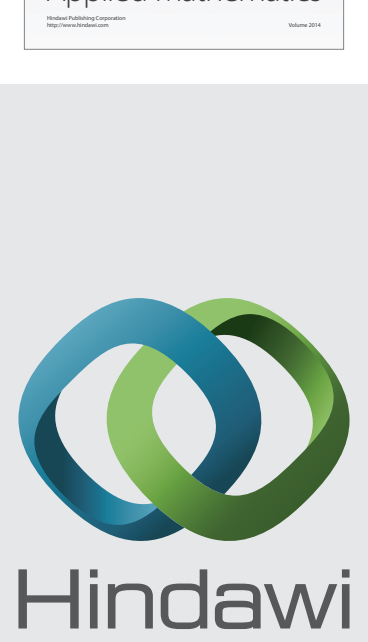

Submit your manuscripts at http://www.hindawi.com
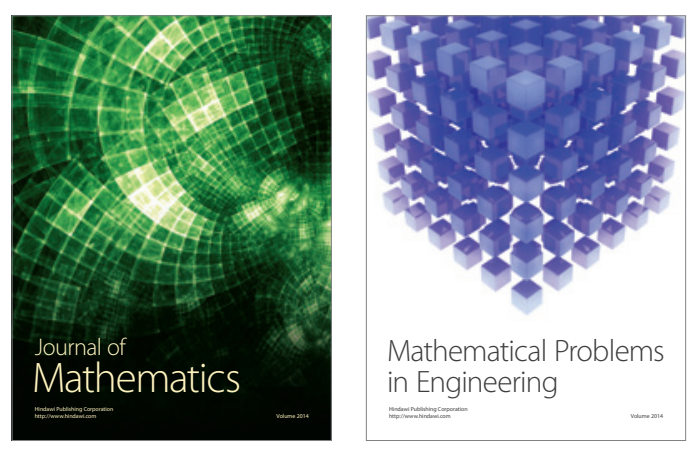

Mathematical Problems in Engineering
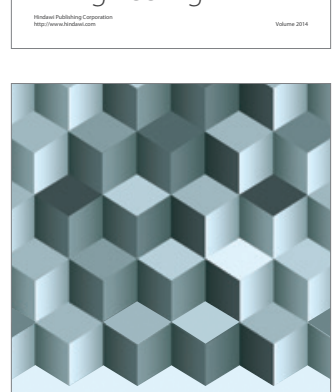

Journal of

Function Spaces
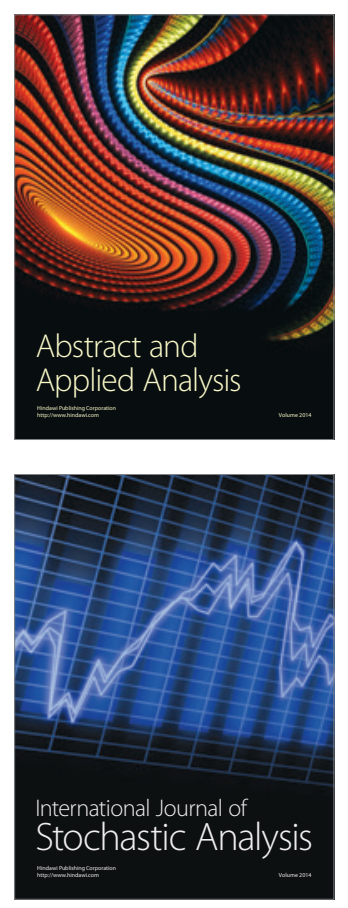

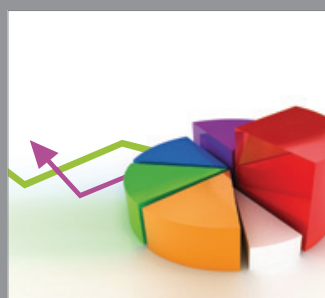

ournal of

Probability and Statistics

Promensencen
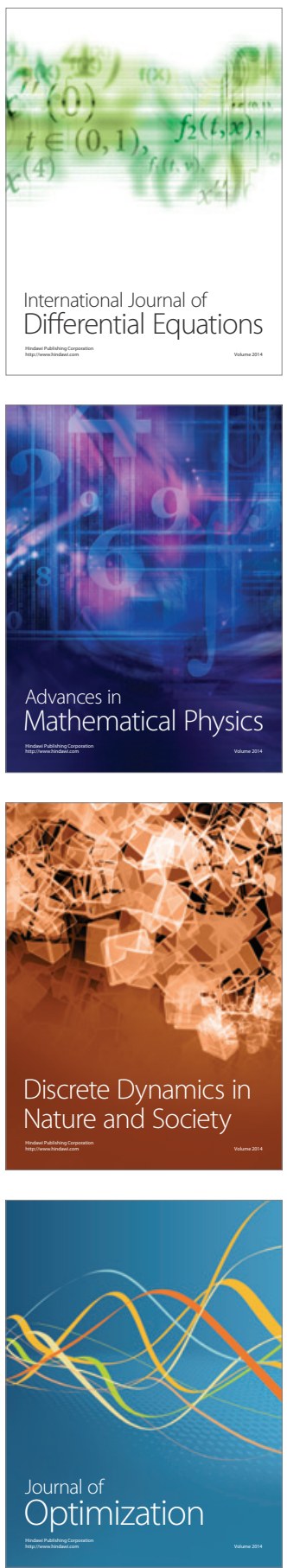